\title{
Impacts of hydroxyvitamin D deficiency on impaired balance function and muscle weakness in young adults
}

\author{
Yu Mori $^{1} \mathbb{1} \cdot$ Toshimi Aizawa $^{1}$
}

Received: 4 August 2021 / Accepted: 4 August 2021 / Published online: 23 August 2021

(c) The Japanese Society Bone and Mineral Research 2021

To the editor

We read with interest the article by Balcanci et al. [1] titled "The relationship between serum hydroxyvitamin D levels and fall risks in young adults". Although the effect of vitamin D administration on the prevention of falls in elderly people with vitamin $\mathrm{D}$ deficiency has been studied, the balance function decline caused by vitamin D deficiency in young adult people has not been studied, and therefore this is a novel study. Balcanci et al. indicated that objective computerized balance function assessment tests showed that even young adults with a 25-hydroxyvitamin $\mathrm{D}(25(\mathrm{OH}) \mathrm{D})$ deficiency had an impaired balance function and an increased risk of falling. In contrast, there was no description of whether young adult patients with $25(\mathrm{OH})$ D deficiency have clinical symptoms such as myalgia, bone pain, and muscle weakness, but we considered it was necessary to describe which cases were measured for $25(\mathrm{OH}) \mathrm{D}$ deficiency.

In this study, balance function was assessed by conventional Berg Balance Test (BBT) and computerized posturography. There was no significant difference in the comparative study in BBT. In contrast, the computerized posturography evaluation showed a significant difference between the low $25(\mathrm{OH}) \mathrm{D}$ group and the normal group. Whether BBT or computerized posturography better reflects the impaired balance function in young adults with low $25(\mathrm{OH}) \mathrm{D}$ needs to be investigated in future studies to measure the actual number of falls and compare with healthy controls.

The balance function is important in preventing falls, but lower limb and trunk muscle strength are also important. Eldecalcitol, an active form of vitamin D, has been reported to promote myocyte differentiation [2]. In addition, there are reports that vitamin D administration improved the balance, muscle strength and the risk of falling in elderly populations [3], while eldecalcitol administration improved only lower limb muscle strength [4]. In this study, we are curious to know whether the group of young adults with low 25(OH)D levels showed obvious muscle weakness in the lower limbs and trunk. The future studies will be expected to reveal more about relationships between vitamin D deficiency and impaired balance function and muscle strength.

\section{References}

1. Balcanci O, Melikoglu MA (2021) The relationship between serum hydroxyvitamin D levels and fall risks in young adults. J Bone Miner Metab. https://doi.org/10.1007/s00774-021-01252-Z

2. Saito H, Kishimoto KN, Mori Y, Okuno H, Tanaka M, Itoi E (2017) A vitamin D analogue, eldecalcitol, enhances expression of fast myosin heavy chain subtypes in differentiated $\mathrm{C} 2 \mathrm{C} 12$ myoblasts. J Orthop Sci 22:345-350. https://doi.org/10.1016/j. jos.2016.12.005

3. Pfeifer M, Begerow B, Minne HW, Suppan K, Fahrleitner-Pammer A, Dobnig H (2009) Effects of a long-term vitamin D and calcium supplementation on falls and parameters of muscle function in community-dwelling older individuals. Osteoporos Int 20:315322. https://doi.org/10.1007/s00198-008-0662-7

4. Saito T, Mori Y, Irei O, Baba K, Nakajo S, Itoi E (2021) Effect of eldecalcitol on muscle function and fall prevention in Japanese postmenopausal women: a randomized controlled trial. J Orthop Sci 26:173-178. https://doi.org/10.1016/j.jos.2020.02.004

Publisher's Note Springer Nature remains neutral with regard to jurisdictional claims in published maps and institutional affiliations.

Yu Mori

yu-mori@med.tohoku.ac.jp

1 Department of Orthopaedic Surgery, Tohoku University Graduate School of Medicine, 1-1 Seiryo-machi, Aoba-ku, Sendai, Miyagi 980-8574, Japan 